\title{
Effect of Autologous Platelet-Rich Plasma (PRP) Treatment on Endometrial Receptivity in ICSI Cases
}

\section{Wafaa Salih Rahmatullah ${ }^{1}$, Manal Taha Al-Obaidi (D) 1, Huda Ali Hussaini (D) 2}

${ }^{1}$ High Institute of Infertility Diagnosis and Assisted Reproductive Technologies, Al Nahrain University, Baghdad, Iraq. wafaarahmatallah1991@gmail.com

${ }^{2}$ College of Medicine, Al Nahrain University, Baghdad, Iraq.

The crosstalk between a receptive endometrium and a functional blastocyst during human embryo implantation is crucial for conception. Because platelets rich plasma (PRP) with concentrated platelets 4-5 times higher than normal, when release of granules containing growth factors including VEGF, TGF, PDGF, IGF, and EGF, these factors involved for sub endometrial angiogenesis and endometrial receptivity. 44 women under the age of 40 were given antagonist ovarian stimulation treatments, the oocytes were harvested utilizing 2-D power doppler ultrasound guidance, then ICSI done for them. On the hCG day all had an intrauterine autologous PRP infusion. Power Doppler is utilized to measure endometrial thickness (EnT), pulsatility index (PI), and resistance index (RI) of sub endometrial arteries, as well as serum levels of VEGF and EGF were measured on the hCG day and ovum pickup (OPU). The ultrasound findings, on the hCG day compared to those of OPU in all women with intrauterine infused autologous PRP were highly significant (HS). The mean EnT, RI, PI, VEGF and EGF at OPU day was lower than that on hCG day in a HS manner, with $(\mathrm{p}<0.001)$, $(\mathrm{p}<0.001)$, $(\mathrm{p}=0.047),(\mathrm{p}<0.001)$, and $(\mathrm{p}<0.001)$ respectively. These characteristics were shown to be significantly and independently associated to intrauterine PRP infusion. After PRP injection, both growth factors serum levels (VEGF and EGF) increased, and ultrasonography sub-endometrial metrics such as EnT, RI, and PI changed as the thickness grew while vascular resistance decreased, and all considered as predictors of endometrial receptivity. 


\section{Introduction}

Reproduction is mandatory for the persistence of all mammalian species. Rendering to World Health Organization (WHO ${ }^{[1]}$ ) "a disease of the reproductive system defined by the failure to achieve a clinical pregnancy after 12 months or more of regular unprotected sexual intercourse," (Szamatowicz and Szamatowicz $\left.{ }^{[2]}\right)$. Implantation failure remains an unsolved issue in assisted reproductive technologies (ART), where good quality embryos are transplanted as a standard of care. What we are dealing with right now is the fact that implantation failure in ART is widespread, and we do not have any evidence-based therapy remedies for it. As a result, practitioners are frequently compelled to recommend treatments that are mostly empirical, based on some biological rationale but with little clinical proof to back them up (Dieamant, et al. ${ }^{[3]}$ ). It is widely known that blastocysts cannot attach to an immature endometrium, resulting in unsuccessful implantation even when "good quality" embryos are transferred (Elsokkary, et al. ${ }^{[4]}$ ). In the early stages of pregnancy, angiogenesis is thought to be a crucial component of proper implantation. Angiogenesis, which occurs predominantly in the uterus, renders the endometrium viable enough to receive the embryo after fertilization. Vascular endothelial growth factor (VEGF) and epidermal growth factors (EGF) are crucial and mandatory required molecular signal for endometrial receptivity as an angiogenesis marker (Liu, et al. ${ }^{[5]}$ ). The terms growth factor and cytokine are occasionally used interchangeably, both are signaling chemicals that connect to specific receptors on their target cells' surfaces, stimulating cell proliferation, differentiation, maturation, or death. Growth factors are key players in angiogenesis, and they have been demonstrated to operate as a critical regulator in the implantation process by acting in an autocrine manner on endometrial epithelial cell adhesion (Bos-Mikich, et al. ${ }^{[6]}$ ). Platelets rich plasma (PRP) stands for plasma platelet concentration acquired by centrifugation of the patient's entire blood. Cytokines and growth factors become bioactive once platelets are activated in PRP and are secreted within 10 
minutes of clotting. VEGF, TGF, PDGF, IGF and EGF are some of these growth factors. (Fioravanti, et al. ${ }^{[7]}$ ). Ultrasound, on the other hand, demonstrates the basal endometrial layer, which is the true interface between the inner myometrium and the endometrium, quite clearly. The majority of the current research focuses on predicting assisted reproductive success rate by measuring midluteal phase endometrial volume and vascular factors. During ART cycles, ultrasound assessment of the uterine cavity is commonly used to determine the thickness, echogenicity, subendometrial resistance, and pulsatility indices of the receptive endometrium (Hou, et al. ${ }^{[8]}$ ). On the day of human chorionic gonadotropin (hCG) is released, a hypoechogenic endometrium with a thickness of more than 7 $\mathrm{mm}$ and low RI and PI is considered a receptive endometrium (Rahmatullah, et al. $\left.{ }^{[9]}\right)$. The existence of arteries inside the endometrium is linked to a thicker endometrium, suggesting that endometrial perfusion and endometrial development are linked. The absence of endometrial-sub endometrial blood flow, on the other hand, is associated by a high uterine artery resistance (Khan, et al. ${ }^{[10]}$ ). In IVF cycles, the resistance index assessed on the day of embryo transfer was much lower in patients who went on to get pregnant compared to those who did not (Maged, et al. ${ }^{[11]}$ ). We aim here to determine whether serum VEGF and EGF levels following PRP injection on the day of ovum pickup can be used as a predictor of endometrial receptivity and pregnancy outcome.

\section{Materials and Methods}

A prospective comparative study was conducted in the High Institute for Infertility Diagnosis and Assisted Reproductive Technologies, Al Nahrain University, Iraq, Baghdad, from October 2019 to April 2021. Forty-four women were included in this study. Before participating in the study, each patient supplied her written informed consent, which was approved by Al Nahrain University's Ethics Committee. They were evaluated twice during the course of the study, and they were not paid for their time. A total of forty-four women were chosen from the High Institute for Infertility Diagnosis and Assisted 
Reproductive Technologies who were having an IVF/ICSI cycle for the study. A complete obstetrical, medical, surgical, and history was required. Assessment of height and weight to determine the body mass index (BMI), clinical and gynecological examinations to rule out any abnormalities, seminal fluid analysis for male partners in accordance with WHO 2010, hormonal analysis (FSH, LH, E2, Testosterone, Prolactin, TSH) for female partners on day 2 of the menstrual cycle, and all patients received antagonist ovarian stimulation. The GnRH antagonist (GnRH ant) protocol is more convenient for the patient with lower overall dose of Gonadotropin and lower cancellation rates used for high and poor responders (Klement, et al. ${ }^{[12]}$ ). When a lead follicle reached a mean diameter of $14 \mathrm{~mm}$, a daily dose of $0.25 \mathrm{mg} \mathrm{GnRH}$ ant. (Cetrorelix acetate for injection $0.25 \mathrm{mg}$ : Cetrotide ${ }^{\circledR}$, Merk, Switzerland) was started and continued until the day of hCG administration (flexible method) (Zhang, et al. ${ }^{[13]}$ ). Follow up of patients done with serial vaginal ultrasound and serum level of estradiol, then accordingly ovum PickUp done. At day of trigger of ovulation, 2D Power Doppler ultrasound of endometrium-zone 2, and serum level of VEGF and EGF measured for all women and had been undergone intrauterine infusion of 0.5-1 $\mathrm{ml}$ of autologous PRP. On the day of ovum PickUp (OPU), again, 2D Power Doppler ultrasound of endometrium-zone 2, and serum level of VEGF and EGF measured for all patients, Transvaginal ultrasound guided Oocyte retrieval done after triggering of ovulation with hCG about 35-36 hrs. And at the day of embryo transfer, usually 2 or 3 days according to the number and grading of embryos, embryo transfer done, then Luteal Phase Support (LPS) was done. On the $14^{\text {th }}$ day after the embryo transfer, a serum B-HCG assay was performed. An ultrasound test was performed subsequently on a lady who had a positive result in order to objectify the presence of 1 or 2 gestational sacs. Blood sampling for VEGF and EGF, at day of trigger and day of OCR, and according to the number and grading of the embryos, patients were prepared for the transfer. The patient's serum was obtained by placing the blood samples in a clean dry plain plastic tube and allowed to 
clot at $37{ }^{\circ} \mathrm{C}$ for 30 minutes prior to centrifugation. The tubes were centrifuged at $5000 \mathrm{rpm}$ for $5 \mathrm{~min}$. All samples were kept in a sterile container labeled with patient's study number. Samples were refrigerated at $-4{ }^{\circ} \mathrm{C}$ until the time of examination. Both kits of VEGF and EGF use Enzyme-Linked Immunosorbent Assay (ELISA) based on biotin double antibody sandwich technology to assay Human Vascular Endothelial Cell Growth Factor A (VEGF-A) and Human Epidermal growth factor (EGF).

\section{Preparation of Platelet Rich Plasma (PRP)}

PRP is made from a sample of the patient's venous blood taken on the day of the hCG trigger. Because a 30-cc venous blood sample provides 3-5 cc of PRP, 5-6 cc of blood is enough to yield $0.5-1 \mathrm{~mL}$ PRP, depending on the individual's baseline platelet count, the device utilized, and the procedure used. After getting written informed consent from the selected women, PRP is produced using a method known as differential centrifugation, in which the acceleration force is regulated to sediment particular cellular components based on differing specific gravity. Before centrifugation, Whole Blood (WB) was collected in tubes containing 3.2 percent sodium citrate solution and stored at $20{ }^{\circ} \mathrm{C}$ to $24{ }^{\circ} \mathrm{C}$. A high-speed centrifuge was used to spin the WB (3000 $\mathrm{g}$ for $6 \mathrm{~min}$.). It develops three layers due of its density: a bottom layer of Red Blood Cells (RBCs), a middle thin layer of platelets and White Blood Cells (WBCs) (buffy coat), and a top layer of Platelet Poor Plasmas (PPPs). The difficult aspect is separating this thin buffy coat layer from the underlying RBC layer. The container's top was emptied of supernatant plasma (PPP). After second spin (3000 $\mathrm{g}$ for $3 \mathrm{~min}$.), 0.4-0.5 $\mathrm{mL}$ of PRP was aspirated and administered into the uterine cavity through an intrauterine insemination (IUI) catheter while the patient was in the lithotomy position and the vulva and vagina were cleaned with normal saline. This procedure is usually preceded by an endometrial (zone-2) ultrasound examination and blood sample for VEGF and EGF levels. 


\section{Results}

\subsection{Demographic}

Characteristics of Infertile Women

The mean age of all enrolled women was $28.93 \pm 5.63$ years, the mean duration of infertility was $7.80 \pm 3.92$ years, $86.4 \%$ were presented with primary infertility and the rest $13.6 \%$ were with secondary infertility, the mean body mass index (BMI) was 27.31 \pm 3.75 $\mathrm{Kg} / \mathrm{m}^{2}$, as shown in Table 1 . The mean serum FSH of all enrolled women was $6.86 \pm 1.90$ $\mathrm{mIU} / \mathrm{mL}$, of LH level was $5.26 \pm 1.51 \mathrm{mIU} / \mathrm{mL}$, of serum estradiol was $33.70 \pm 13.05 \mathrm{pg} / \mathrm{mL}$, of serum prolactin was $17.81 \pm 8.87 \mathrm{ng} / \mathrm{mL}$, and of serum TSH was $1.95 \pm 1.48 \mathrm{mIU} / \mathrm{L}$. While the mean serum estradiol of enrolled women on the day of hCG was $1418.80 \pm 616.31 \mathrm{pg} / \mathrm{mL}$, the mean total dose of gonadotropin of all enrolled women was $1698.3 \pm 647.90 \mathrm{u}$, the mean duration of stimulation of all enrolled women was $9.27 \pm 1.56$ days, and the mean total oocyte count of all enrolled women was $11.80 \pm 6.01$, The mean number of transferred embryos of all enrolled women was $2.82 \pm 0.97$, as shown in Table 2.
4.2. Ultrasound findings, Doppler biophysical profile and serum growth factor levels in all enrolled women at day of trigger and day of Ova PickUp

According to the results shown in Figure 1 that compares the ultrasound parameters, biophysical profile, and serum growth factor levels in the women on the day of trigger to those on the day of ovum pickup, the mean endometrial thickness (EMT) on the day of OPU was higher than that on the day of trigger in a highly significant manner, $10.38 \pm 1.56 \mathrm{~mm}$ versus $8.58 \pm 1.71 \mathrm{~mm}$, respectively $(\mathrm{p}<$ 0.001), as shown in Figure 1 (a). While the mean resistance index $(\mathrm{RI})$ on the day of OPU was lower than that on the day of trigger in a highly significant manner, $0.49 \pm 0.08$ versus $0.62 \pm 0.09$, respectively ( $\mathrm{p}<0.001$ ), as shown in Figure 1 (b). The mean pulsatility index (PI) on the day of OPU was lower than that on the day of trigger also in a significant manner, $0.78 \pm 0.55$ versus $0.82 \pm 0.24$, respectively $(\mathrm{p}=0.047)$, as shown in Figure 1 (c). The mean VEGF on the day of OPU was higher than that on the day of trigger in a highly significant 
manner, $186.53 \pm 60.21$ versus $144.42 \pm 47.80$, respectively $(\mathrm{p}<0.001)$, as shown in Figure 1 (d). The mean EGF on the day of OPU was higher than that on the day of trigger in a highly significant manner, 88.76 \pm 40.614 versus 60.92 \pm 39.10 , respectively $(p<0.001)$, as shown in Figure 1 (e). The rate of pregnancy was $31.8 \%$, and it was good in comparison with the ratio of natural conception, and it is considered satisfied regarding all percentages in the world, Figure 1 (f).

\section{Discussion}

Successful implantation necessitates the presence of a viable embryo, a receptive endometrium, adequate embryo endometrial cross-talk, and sufficient mother immune protection. Despite advances in ART, the rates of implantation and pregnancy have remained stagnant.

5.1. Ultrasound Parameters in PRP intrauterine infused women at day of hCG and day of $O P U$

a. Endometrial Thickness
The EMT of PRP group women differed significantly between the day of hCG and the day of OPU in this study; it was higher after PRP intrauterine infusion, and the difference was attributed to the favorable effect of autologous PRP. In a study of over 2600 infertile women, investigates the role of PRP in enhancing endometrial receptivity, researchers discovered that $38.5 \%$ of cycles had a $10 \%$ plateau level of EMT (no change after hCG trigger) and $37.2 \%$ of cycles had a $>10 \%$ increase group, while fewer cycles $(24.2 \%)$ had a $>10 \%$ decrease in EMT more than 2-3 days after trigger (Huang, et al. ${ }^{[14]}$ ). Several treatments have been taken in an attempt to improve EMT, but the outcomes have been mixed. Platelets carry a large number of growth factors that aid tissue repair in the local area. (Larson, et al. ${ }^{[15]}$ ) had been employing platelet-derived growth factor (PDGF) to successfully develop bovine embryos throughout the fourth cell cycle since 1992. This was the beginning of an evolution in reducing abortion rate by increasing EMT. Between 2016 and 2017, 72 women with a history of cancelled frozen embryo transfer 
Table (1): Demographic parameters of patients enrolled in the study

\begin{tabular}{|c|c|}
\hline Characteristic & Study group $(n=44)$ \\
\hline \multicolumn{2}{|c|}{ Age (vears) } \\
\hline Mean \pm SD & $28.93 \pm 5.63$ \\
\hline Range & $18-40$ \\
\hline \multicolumn{2}{|c|}{ Duration of infertility (years) } \\
\hline Mean \pm SD & $7.80 \pm 3.92$ \\
\hline Range & $1-17$ \\
\hline \multicolumn{2}{|c|}{ BMI $\left(\mathrm{kg} / \mathrm{m}^{2}\right)$} \\
\hline Mean \pm SD & $27.31 \pm 3.75$ \\
\hline Range & $20.2-36.7$ \\
\hline \multicolumn{2}{|c|}{ Type of infertility } \\
\hline Primary, $n(\%)$ & $38(86.4 \%)$ \\
\hline Secondary, $n(\%)$ & $6(13.6 \%)$ \\
\hline
\end{tabular}

Table (2): Hormonal profile of patients enrolled in the study

\begin{tabular}{|c|c|}
\hline Characteristic & Study group $(n=44)$ \\
\hline \multicolumn{2}{|c|}{ FSH $(\mathrm{mIU} / \mathrm{ml})$} \\
\hline Mean \pm SD & $6.86 \pm 1.90$ \\
\hline Range & $3.15-10.7$ \\
\hline \multicolumn{2}{|c|}{$\mathrm{LH}(\mathrm{mIU} / \mathrm{ml})$} \\
\hline Median (IQR) & $5.26 \pm 1.51$ \\
\hline Range & $3.08-10.4$ \\
\hline \multicolumn{2}{|c|}{ Estradiol $(\mathrm{pg} / \mathrm{ml})$} \\
\hline Mean \pm SD & $33.70 \pm 13.05$ \\
\hline Range & $4.7-69$ \\
\hline \multicolumn{2}{|c|}{ Prolactin (ng/ml) } \\
\hline Mean \pm SD & $17.81 \pm 8.87$ \\
\hline Range & $2.5-42$ \\
\hline \multicolumn{2}{|c|}{ TSH mIU/L } \\
\hline Mean \pm SD & $1.95 \pm 1.48$ \\
\hline Range & $0.39-7.3$ \\
\hline
\end{tabular}

$\boldsymbol{n}$ : number of cases; SD: standard deviation; BMI: body mass index IQR: inter-quartile range; FSH: follicle stimulating hormone; LH: Luteinizing hormone; TSH: thyroid stimulating hormone;

Rahmatullah, et al. http://doi.org/10.28969/IJEIR.v10.i2.r6 


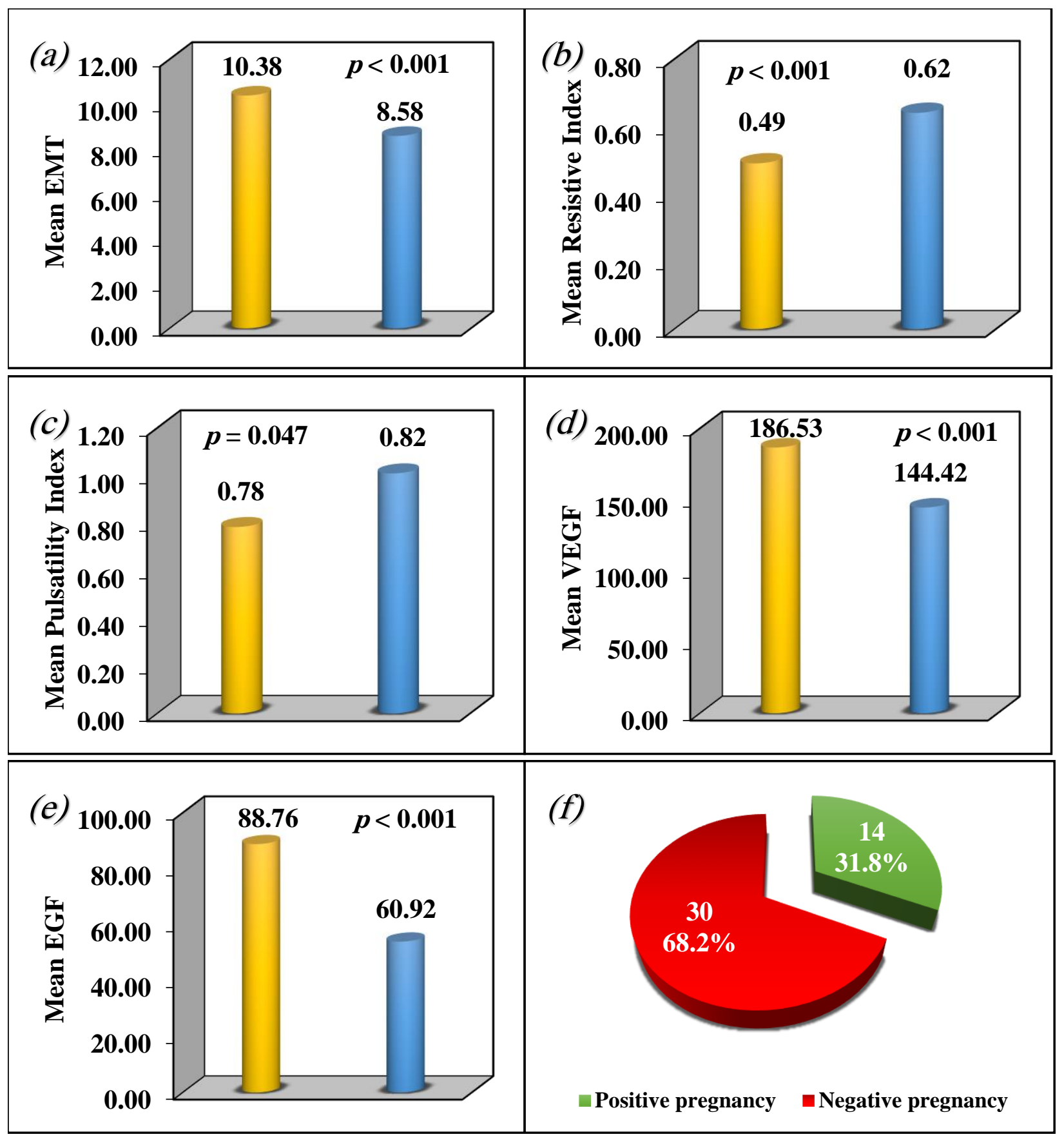

Figure (1): Comparison of ultrasound parameters between day of ovulation trigger (in blue accent 5 color) and day of ova pickup (in gold accent 4 color) (a) mean EMT (b) mean RI (c) mean PI (d) mean VEGF (e) mean EGF (f) pregnancy rate 
cycles due to inadequate endometrial development $(7 \mathrm{~mm})$ despite standard treatments were assessed for eligibility to enter the study with the help of another set of researchers. They suggested that intrauterine PRP infusions could help in endometrial development, expansion, and implantation. EMT increased 48 hours after the first PRP and reached $>7 \mathrm{~mm}$ after the second PRP. All patients had embryo transfer, and according to their research, PRP was helpful in endometrial enlargement in patients with refractory thin endometrium, with $12 \%$ of women participated in the study achieving pregnancy (Nazari, et al. ${ }^{[16]}$ ). PRP intrauterine infusion was recommended in two recent research for two innovative ART techniques. These researches indicated the beneficial benefits of PRP in Asherman's syndrome regeneration and the good effects of PRP in improving pregnancy outcomes in repeated implantation failure (Aghajanova, et al. ${ }^{[17]}$, Nazari, et al. ${ }^{[18]}$ ), and all of these studies coincided with ours. The use of autologous PRP as a potential approach of increasing EMT in women with refractory endometrium was also mentioned in a review article by (Garcia-Velasco, et al. $\left.{ }^{[19]}\right)$. It would assist to reduce the number of cycle cancellations, as well as the financial and psychological costs of recurrent cycle cancellations.

\section{b. Resistance and Pulsatility Indices}

In the current study, RI and PI in PRP intrauterine infused women were at day of ovulation trigger higher than that at day of OPU, so both decline after using autologous PRP intrauterine infusion. When they analyzed uterine artery fluxometry (resistance and pulsatility index), several writers agreed with our findings and found that the statistical difference values were lower in the implantation group than in the nonimplantation group. When they looked at the mean EMT before PRP, they found that it grew significantly after PRP, and that endometrial vascularity increased statistically significantly. Pre-PRP, the mean PI uterine artery was higher than after PRP (Motwani and Sharma ${ }^{[20]}$ ). In other study, (Garcia-Velasco, et al. ${ }^{[19]}$ ) advocated for the importance of PRP in increasing the number of vascular signals seen 
on Power Doppler, reaching zones 3 and 4 of the endometrium, after intrauterine autologous PRP infusion on a group of women undergoing frozen embryo transfer cycles with suboptimal endometrial pattern as assessed by transvaginal ultrasound and Power Doppler. On the contrary to our findings, some researchers discovered that elevated pulsatility index (PI) and resistive index (RI) of the uterine arteries were associated with low pregnancy and implantation rates, and that adequate Doppler velocimetry of the uterine arteries was slightly specific for predicting gestation, they measured endometrial flow impedance in 156 patients on the day of OPU and found no change in PI, RI, or S:D ratio measures between women who conceived and those who did not (Singh, et al. ${ }^{[21]}$ ). While PRP administration in infertile women undergoing frozen embryo transfer cycles with suboptimal endometrium induced a significant increase in vascularity, as measured by the number of vascular signals seen on Power Doppler, reaching even the zones 3 and 4 of the endometrium, according to (Bos-Mikich, et al. $\left.{ }^{[6]}\right)$.

\section{c. Serum Growth Factors Level}

According to various studies, endometrial vascularity is an important metric to consider when evaluating the endometrium's implantation capability. The levels of VEGF and EGF were higher in women on the day of OPU than on the day of ovulation trigger in our study, which was attributed to the proliferative and progressive effect of PRP on the endometrium. It is possible that a significant lack of these angiogenesis-related signal molecules causes the endometrium to form abnormally and remain thin. Most scientists, including (Alfer, et al. ${ }^{[22]}$ ), believed that a lack of angiogenetic development could explain the endometrial component that causes infertility. In addition, immunohistochemistry demonstrated that VEGF expression in recurrent spontaneous abortions patients was much lower than in normal pregnant women. The role of VEGF expression in the pathophysiology of repeated spontaneous abortions was investigated in a study by (Zhu, et al. ${ }^{[23]}$ ) in order to confirm the negative effects of VEGF insufficiency on the immune 
system. As a result, PRP is required for implantation as a source of growth factors.

\section{Conclusions}

Five characteristics were shown to be significantly and independently associated to intrauterine PRP infusion. Endometrial thickness, RI, PI, VEGF and EGF are the variables in question. After PRP injection, both growth factors serum levels increased, as did ultrasonography sub-endometrial metrics showed changes in endometrial thickness, RI, and PI, as the thickness grew vascular resistance decreased, and all considered as predictors of endometrial receptivity.

\section{Acknowledgment}

We would like to acknowledge Al Nahrain University, Baghdad, Iraq.

\section{Funding}

This work was funded by the corresponding author.

\section{Author Contribution}

Rahmatullah, WS, performed the study, examined and reviewed results, and manuscript writing with the help and supervision of Al-Obaidi, MT, and Huda, HA.

\section{Conflict of Interest}

The authors declare no conflict of interest.

\section{Ethical Clearance}

The study was approved by the Ethical Approval Committee.

\section{References}

[1] World Health Organization. WHO laboratory manual for the examination and processing of human semen, $5^{\text {th }}$ ed. 2010 World Health Organization. [Online article link]

[2] Szamatowicz M, Szamatowicz J. Proven and unproven methods for diagnosis and treatment of infertility. Advances in Medical Sciences. Elsevier BV; 2020 Mar;65(1):936. [Online article link]

[3] Dieamant F, Vagnini LD, Petersen CG, Mauri AL, Renzi A, Petersen B, et al. New therapeutic protocol for improvement of endometrial receptivity (PRIMER) for patients with recurrent implantation failure 
(RIF) - A pilot study. JBRA Assisted Reproduction. GN1 Genesis Network; 2019. [Online article link]

[4]Elsokkary M, Eldin AB, Abdelhafez M, Rateb A, Samy M, Eldorf A, et al. The reproducibility of the novel utilization of five-dimensional ultrasound and power Doppler in the prediction of endometrial receptivity in intracytoplasmic sperminjected women: a pilot prospective clinical study. Archives of Gynecology and Obstetrics. Springer Science and Business Media LLC; 2018 Dec 19;299(2):551-8. [Online article link]

[5] Liu H, Chen Y, Yan F, Han X, Wu J, Liu X, et al. Ultrasound Molecular Imaging of Vascular Endothelial Growth Factor Receptor 2 Expression for Endometrial Receptivity Evaluation. Theranostics. Ivyspring International Publisher; 2015;5(2):206-17. [Online article link]

[6]Bos-Mikich A, Ferreira MO, de Oliveira R, Frantz N. Platelet-rich plasma or bloodderived products to improve endometrial receptivity? Journal of Assisted Reproduction and Genetics. Springer
Science and Business Media LLC; 2019 Jan 4;36(4):613-20. [Online article link]

[7]Fioravanti C, Frustaci I, Armellin E, Condò R, Arcuri C, Cerroni L. Autologous blood preparations rich in platelets, fibrin and growth factors. ORAL \& implantology (Rome). 2016 Jul 23;8(4):96-113. [Online article link]

[8] Hou Z, Zhang Q, Zhao J, Xu A, He A, Huang X, et al. Value of endometrial echo pattern transformation after hCG trigger in predicting IVF pregnancy outcome: a prospective cohort study. Reproductive Biology and Endocrinology. Springer Science and Business Media LLC; 2019 Sep 5;17(1). [Online article link]

[9] Rahmatullah WS, Al-Obaidi MT, AL-Saadi WI, Selman MO, Faisal GG. Role of Vascular Endothelial Growth Factor (VEGF) and Doppler Sub-endometrial Parameters as Predictors of Successful Implantation in Intracytoplasmic Sperm Injection (ICSI) Patients. Siriraj Medical Journal. Faculty of Medicine Siriraj Hospital, Mahidol University; 2020 Jan 1;72(1):33-40. [Online article link] 
[10] Khan MS, Shaikh A, Ratnani R. Ultrasonography and Doppler Study to Predict Uterine Receptivity in Infertile Patients Undergoing Embryo Transfer. The Journal of Obstetrics and Gynecology of India. Springer Science and Business Media LLC; 2015 Sep 8;66(S1):377-82. [Online article link]

[11] Maged AM, Kamel AM, Abu-Hamila F, Elkomy RO, Ohida OA, Hassan SM, et al. The measurement of endometrial volume and sub-endometrial vascularity to replace the traditional endometrial thickness as predictors of in-vitro fertilization success. Gynecological Endocrinology. Informa UK Limited; 2019 Apr 21;35(11):949-54. [Online article link]

[12] Hershko Klement A, Berkovitz A, Wiser A, Gonen O, Amichay K, Cohen I, et al. GnRH-antagonist programming versus GnRH agonist protocol: a randomized trial. European Journal of Obstetrics \& Gynecology and Reproductive Biology. Elsevier BV; 2015 Feb;185:170-3. [Online article link]
[13] Zhang D, Xia L, Xu H, Chen Q, Jin B, Zhang A, et al. Flexible Low-Dose GnRH Antagonist Protocol Is Effective in Patients With Sufficient Ovarian Reserve in IVF. Frontiers in Endocrinology. Frontiers Media SA; 2018 Dec 19;9. [Online article link]

[14] Huang J, Lin J, Cai R, Lu X, Song N, Gao H, et al. Significance of endometrial thickness change after human chorionic gonadotrophin triggering in modified natural cycles for frozen-thawed embryo transfer. Annals of Translational Medicine. AME Publishing Company; 2020 Dec;8(23):1590-1590. [Online article link] [15] Larson RC, Ignotz GG, Currie WB. Platelet derived growth factor (PDGF) stimulates development of bovine embryos during the fourth cell cycle. Development. $1992 \mathrm{Jul} ; 115(3): 821-6$. [Online article link]

[16] Nazari L, Salehpour S, Hoseini S, Zadehmodarres S, Azargashb E. Effects of autologous platelet-rich plasma on endometrial expansion in patients undergoing frozen-thawed embryo transfer: A double-blind RCT. International Journal of Reproductive BioMedicine. Knowledge 
E; 2019 Jul 29; 443-448. [Online article $\underline{\text { link] }}$

[17] Aghajanova L, Houshdaran S, Balayan $\mathrm{S}$, Irwin $\mathrm{J}$, Huddleston $\mathrm{H}$, Giudice $\mathrm{L}$. Platelets for endometrial regeneration: a novel approach. Fertility and Sterility. Elsevier BV; 2016 Sep;106(3):e82. [Online article link]

[18] Nazari L, Salehpour S, Hoseini S, Zadehmodarres S, Ajori L. Effects of autologous platelet-rich plasma on implantation and pregnancy in repeated implantation failure: A pilot study. International Journal of Reproductive BioMedicine. 2016 Oct;14(10):625-628. [Online article link]

[19] Garcia-Velasco JA, Acevedo B, Alvarez C, Alvarez M, Bellver J, Fontes J, et al. Strategies to manage refractory endometrium: state of the art in 2016. Reproductive BioMedicine Online. Elsevier BV; 2016 May;32(5):474-89. [Online article link]

[20] Motwani Y, Sharma R. To study the effect of autologous platelet-rich plasma instillation on suboptimal endometrium and pregnancy outcomes in infertile women undergoing frozen embryo transfer cycle. Fertility Science and Research. Medknow; 2020;7(2):141. [Online article link]

[21] Singh N, Bahadur A, Mittal S, Malhotra N, Bhatt A. Comparative analysis of endometrial blood flow on the day of hCG by 2D Doppler in two groups of women with or without genital tuberculosis undergoing IVF-ET in a developing country. Archives of Gynecology and Obstetrics. Springer Science and Business Media LLC; 2010 Aug 6;283(1):115-20. [Online article link]

[22] Alfer J, Happel L, Dittrich R, Beckmann M, Hartmann A, Gaumann A, et al. Insufficient Angiogenesis: Cause of Abnormally Thin Endometrium in Subfertile Patients? Geburtshilfe und Frauenheilkunde. Georg Thieme Verlag KG; 2017 Jul;77(07):756-64. [Online article link]

[23] Zhu Y, Lu H, Huo Z, Ma Z, Dang J, Dang W, et al. MicroRNA-16 inhibits fetomaternal angiogenesis and causes recurrent spontaneous abortion by targeting vascular 
endothelial growth factor. Scientific Reports. Springer Science and Business Media LLC; 2016 Oct 17;6(1). [Online article link]

Authors at OrcID

Manal T. Al-Obaidi

https://orcid.org/0000-0001-

8722-3159

Huda A. Hussaini (D)

https://orcid.org/0000-0002-

$\underline{5544-8334}$

\section{Peer Review Information}

Double-Blind Peer Review in which both authors and reviewers does not know each other.

This work was reviewed by

Asst. Prof. Dr. Nadia Al-Hilli

Asst. Prof. Dr. Lubna Amer Al-Anbari

\section{Editorial Policy}

The editorial policy at IJEIR ensured that this article fit the standards of scientific publications.

This work was copyedited by

$\underline{\text { Dr. Taif Alawsi }}$

\section{Authors Biographies}

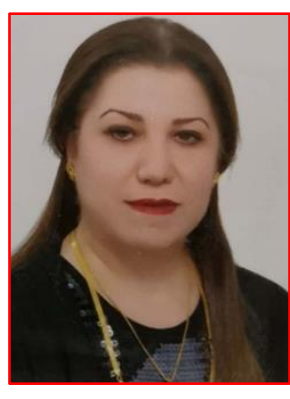

Dr. Wafaa Salih Rahmatullah

She received her M.B.Ch.B., from the College of Medicine, the University of Al-Mustansiriya in 1991. Her Diploma of Gynecology and Obstetrics (D.G.O.), College of Medicine, the University of Baghdad in 2002. Her master in Clinical Infertility and Assisted Reproductive Technique AlNahrain University in 2017 and in the same institute received her Ph.D. in Clinical Infertility in 2021. She is a member of the Iraqi Medical Association since 1991. Since 1993 she has occupied many positions in different institutions being a specialist since 2002 and developing her career actively. She is actively involved in many scientific and research courses and received more than 13 certificates both on the national and international levels. She has been lecturing since 2003 to many different institutions including Al-Furat Al-Awsit Technical University, Al-Semawa Maternity Hospital, Al-Semawa Maternity Hospital, and Al-Dewaniya Teaching Maternity Hospital.

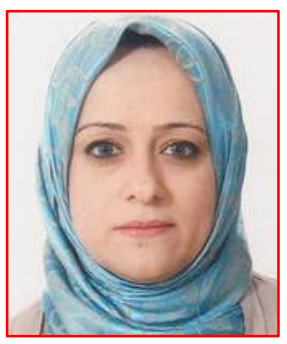

\section{Dr. Manal T. Al-Obaidi}

She received the M.B.CH.B. from AlMustansirya University, the High diploma (equivalent to MSc.) in Assisted Reproductive Technology, Ph.D. in reproductive physiology in 1997, 2003, and 2013 respectively. She occupied several academic positions including the head of the clinical reproductive physiology department and currently, she is the dean of the High Institute of Infertility Diagnosis and Assisted Reproductive 
Technologies, Al Nahrain University. She published more than 33 articles both local and international.

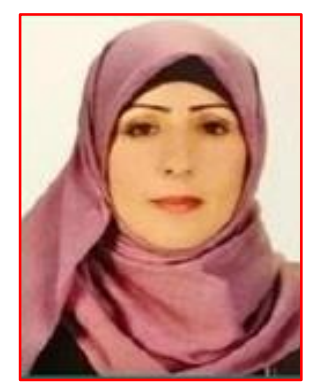

\section{Dr. Huda Ali Hussaini}

She received the M.B.Ch.B: Babylon University, College of Medicine, in 2000. The diploma in Radiology from the University of Baghdad in 2007. She is a fellow of the Iraqi Board for Medical Specialization, Council of Radiology since 2008. Currently, she is an assistant professor. Al Nahrain University, College of Medicine, department of surgery (specialist radiologist). She is a member of staff in the high Institute of infertility diagnosis and ARTS. Including the ultrasound department in the institute since 2018. She has Participations in a lot of annual meetings, medical conferences. Seminars, continuous learning, and thesis discussion. She is a member of the Iraqi medical association, the Iraqi Society of Radiologists and medical imaging, the Iraqi Journal of Embryos infertility researches, European Society for Hybrid, Molecular, and translation imaging. She published more than 15 articles and has supervised many students.

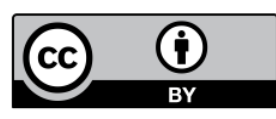

(C) 2021 Author(s)

This article is licensed under a Creative Commons Attribution 4.0 International License, which permits use, sharing, adaptation, distribution and reproduction in any medium or format, as long as you give appropriate credit to the original author(s) and the source, provide a link to the Creative Commons license, and indicate if changes were made.

http://creativecommons.org/licenses/by/4.0/. 